\title{
DimENSIONAMIENTO DE FLOTA EN LAS OPERACIONES DE CARGUÍO Y TRANSPORTE USANDO MODELOS DE SIMULACIÓN DE SISTEMAS
}

\author{
Osmar Lins de Noronha \\ Grupo Pirineus. Goiás, Brasil \\ sac@grupopirineus.com.br \\ Alcides Eloy Cano Nuñez / Adeline F. dos Reis \\ canoalcides@hotmail.com / delinereis@yahoo.com.br \\ Universidade Federal de Goiás. Goiás, Brasil \\ Carlos Enrique Arroyo Ortiz \\ Universidade Federal de Ouro Preto. Minas Gerais, Brasil \\ carroyo@ufop.edu.br
}

\section{Resumen}

El estudio aborda aspectos que deben ser considerados en el dimensionamiento de equipamiento de carguío y transporte de material (mineral y desmonte) en una mina a cielo abierto localizada en el centro-oeste de Brasil. Este proceso es complejo ya que está involucrada en el análisis una significativa cantidad de variables que limitan las actividades intrínsecas a las operaciones de carguío y transporte, tales como: tasas de producción por unidad de tiempo, características físico-mecánicas del material, distancias de transporte, topografía, diseño de accesos, vías de transporte, ciclos y rendimiento de la flota (camión, pala); adicionalmente, existen otras variables externas que también condicionan la normal operación, dentro las cuales podemos citar lluvia, nieve, neblina y movimiento sísmico. El trabajo tiene como finalidad dimensionar la flota de los equipos de carguío y transporte que atienda el requerimiento de planta de procesamiento de manera eficiente.

Palabras clave: dimensionamiento de flota, análisis estocástico, análisis determinístico

\section{Abstract}

\section{Fleet sizing in loading and hauling operations using systems simulation models}

The present study addresses aspects that must be considered in the sizing of the equipment for material loading and haulage (ore and waste) in an open-pit mine in the Central-West Region of Brazil. This is a complex process because a significant number of variables that limit the activities intrinsic to the loading and hauling operations are involved in the analysis. Such variables are: production rates per unit of time, physical-mechanical characteristics of the material, hauling distances, topography, access design, hauling routes, cycles, and fleet performance (truck, shovel). Additionally, there are other external variables that also condition a normal operation, such as rain, snow, fog and seismic movement. The purpose of this study is to size the fleet of the loading and hauling equipment that fulfills the processing plant requirements in an efficient manner.

Keywords: fleet sizing, stochastic, deterministic 


\section{Introducción}

Los costos en las operaciones de carguío y transporte de mineral y desmonte representan una suma considerable en los costos de producción del mineral. Por consiguiente, el dimensionamiento adecuado de estas operaciones es de extrema importancia a fin de reducir dichos costos. Pinto y Saliby (1999) sostienen que la extracción del mineral a cielo abierto se caracteriza por ser una actividad de alto costo de inversión y el comportamiento estocástico del sistema la hace más compleja, así como también cualquier toma de decisión.

De acuerdo con Koppe (2007), pocas minas consiguen armonizar la relación entre la producción requerida y la capacidad de los equipamientos.

Para definir el tipo de equipamiento y sistema que será requerido, deben considerarse y evaluarse variables tales como: capacidad real de los equipos, distancia de transporte, topografía del terreno, infraestructura disponible en la región, interferencias con el medio ambiente, economía, entre otros (Silva, 2009).

Este estudio de dimensionamiento de flota de carguío y transporte vía simulación fue realizado con la información de una mina de caliza localizada en el centro-oeste brasileño. La mina en cuestión es productora de material para la industria de construcción, agrícola, industria de tintas y fibrocementos. Con los resultados obtenidos será posible proponer alternativas y estrategias de producción que permitan atender los requerimientos de la planta concentradora.

Esta investigación tuvo como objetivo principal realizar un apropiado dimensionamiento de flota a través de técnicas avanzadas de programación estocástica. De este modo se podía abordar el problema real usando modelos de simulación, cuyo propósito es evaluar los escenarios de producción. También se buscó predecir el comportamiento del sistema de carguío y transporte de materiales y analizar la utilización de los equipamientos para determinar de manera objetiva el tamaño de la flota que atiende los requerimientos de producción exigidos por la planta. Finalmente, esta investigación se propuso realizar una comparación entre dimensionamiento analítico versus estocástico y, de este modo, obtener mayores elementos de análisis para una toma de decisiones más acertada.

\section{Metodología}

Para el abordaje y entendimiento del problema de dimensionamiento de equipamiento de carguío y transporte se realizó una revisión bibliográfica relacionada con carguío y transporte de mineral y dimensionamiento de flota. Seguidamente fue necesario definir los lineamientos y conocimientos técnicos necesarios para estructurar las actividades, las tareas a ser realizadas por el equipo e identificar las variables (tiempos, distancias, operaciones de 
equipamientos) y parámetros que intervienen en el problema. Posteriormente se establecieron modelos analíticos y estocásticos que representan el problema. Finalmente, se utilizó software como AutoCAD 2014, Mathcad 14.0, Arena 14.0, Excel 2013 y Word 2013 para encontrar los resultados y elaborar los informes correspondientes.

\section{Revisión de literatura de operaciones de carguío y transporte de mineral}

Las operaciones de carguío y transporte de mineral y desmonte son actividades primarias concernientes a los trabajos de extracción mineral propiamente dicha; la importancia de esta actividad se debe al hecho de formar parte de la cadena productiva del bien mineral. Las excavadoras son equipamientos principales usados en las tareas de carguío y están designadas para cumplir actividades de remoción, carga y descarga del material. Seguidamente, después de que las excavadoras hayan llenado la tolva de los camiones con material rocoso fragmentado, estos últimos transportan la carga a la planta de tratamiento o pila de desmonte, o bien el material podría ser llevado a otras áreas como destino final (Dessureault, 2009).

Los camiones transportan el material en sus tolvas y, según su valor económico, es descargado en planta o pila de desmonte (Bise, 2003). En la figura 1 se muestran las actividades de carguío y transporte de material y se describe gráficamente el ciclo integrado de la producción.

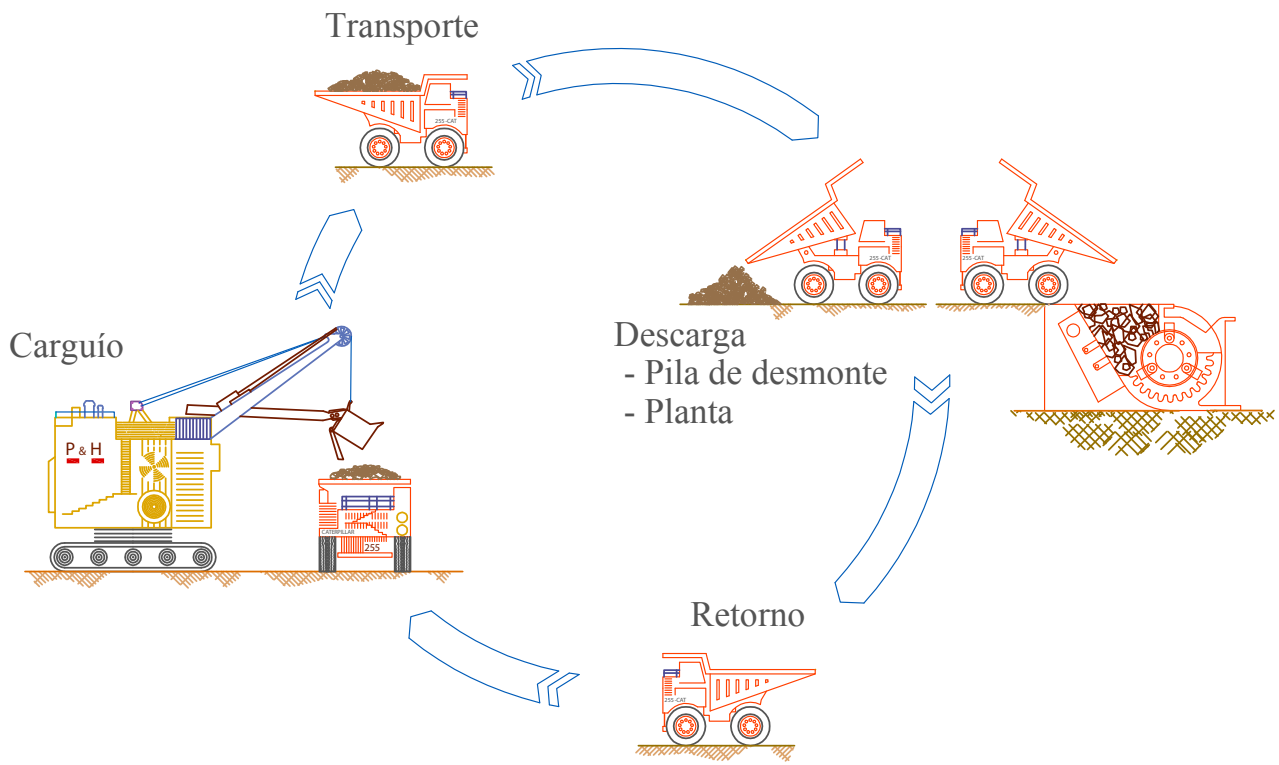

Figura 1. Ciclo de carguío y transporte de mineral o desmonte

Elaboración propia 


\section{Dimensionamiento de la flota de carguío y transporte de mineral}

\subsection{Producción en la mina Calcário Ouro Branco}

La mina tiene cuatro frentes de explotación: Osvaldo, Grande, Mandacaru y Antônio Ferreira. De cada uno de los frentes de explotación son extraídas materias primas para la producción de cinco derivados: tintas, textura, ración animal, fibrocemento y corrector de acidez del suelo para agricultura. De los cinco productos, solo el corrector de acidez es producido por la propia compañía minera; los otros cuatro son producidos por la mina Fillercal Mineração e Comércio (Grupo Pirineus, 2013). La tabla 1 presenta las unidades mineras y las cantidades extraídas, así como los productos de cada una de ellas.

Tabla 1. Producción planeada y programada en cada unidad

\begin{tabular}{|c|c|c|c|c|}
\hline \multirow[b]{2}{*}{ Origen-destino } & \multicolumn{4}{|c|}{ Production } \\
\hline & Ene-abr (2016) t/mes & May-nov (2016) t/mes & $2017 \mathrm{t} / \mathrm{mes}$ & 2017 t/día \\
\hline Osvaldo-planta Ouro Branco (a) & --------- & 21400 & 15000 & 625 \\
\hline Grande-planta Ouro Branco (a) & ------- & 21400 & 15000 & 625 \\
\hline Mandacaru-planta Fillercal (b) & 900 & 900 & 4500 & 188 \\
\hline Antônio Ferreira-planta Fillercal (c) & 2300 & 2300 & 3000 & 125 \\
\hline Antônio Ferreira-clasificación & 8900 & 8900 & 18720 & 780 \\
\hline Clasificación-planta Fillercal (b) & 8900 & 8900 & 18720 & 780 \\
\hline Planta Ouro Branco-pila de desmonte & -------- & 10300 & 10000 & 417 \\
\hline Total anual & 623700 & & 1019280 & \\
\hline
\end{tabular}

(a) insumos para agricultura, (b) insumos para ración animal, (C) insumos para textura, pinturas Fuente: datos facilitados por la Gerencia del Grupo Pirineus

\subsection{Dimensionamiento determinístico de la flota de carguío y transporte de mineral}

Para dimensionar los equipos de carguío y transporte es importante revisar el conocimiento técnico referente a las características de cada equipo de carga y transporte.

Sweigard (2002) reúne las principales características que deben ser consideradas en una selección de equipamientos; además se refiere a estudios de diversos autores que han publicado en el área de transporte de materiales.

Sweigard detalla 11 criterios: 1) producción, 2) tasa de producción, 3) disponibilidad, 4) utilización, 5) eficiencia, 6) productividad, 7) capacidad, 8) capacidad nominal, 9) factor de esponjamiento, 10) factor de llenado de la cuchara y 11) ciclo. El autor indica que la utilización 
está condicionado por: a) características del material, b) supervisión en el trabajo, c) tiempo de espera en zona de descarga (pila de desmonte o planta), d) falta de camión, e) destreza del operador, f) interrupciones para limpieza del frente de trabajo (limpieza de plaza), g) calidad de la fragmentación, h) capacidad de la cuchara del equipo de carguío e i) pequeñas interrupciones debido a defectos mecánicos que generalmente son imprevistos de mantenimiento.

Las operaciones de carguío en la mina Calcário Ouro Branco son realizadas con cuatro excavadoras CAT 336D para los frentes de Osvaldo, Mandacaru y Antônio Ferreira. Además, dos palas CAT 950H, son usadas en la carga de mineral clasificado, y otra en la carga de desmonte proveniente de la planta en la mina Ouro Branco. El ciclo de trabajo de una excavadora se divide en cuatro actividades realizadas secuencialmente: carguío, maniobra con carga, descarga y maniobra sin carga.

Noronha (2016) determinó a través de informaciones de campo y análisis estadístico que el ciclo de la excavadora CAT 336D es de 0,28 minutos, y de 0,53 minutos el de la excavadora CAT $950 \mathrm{H}$ (tales tiempos son valores medios). El transporte de mineral en la mina Calcário Ouro Branco es realizado por medio de volquetes Volvo VM330 de 28,8 t. Estos vehículos realizan seis rutas (circuitos) para el transporte del mineral, una ruta para el desmonte y adicionalmente dos rutas de retorno de pila de desmonte para transporte de mineral.

Los volquetes trabajan en ruta dinámica; por lo tanto, los equipamientos no son exclusivos para una ruta específica. La empresa trabaja dos turnos por día, cada turno de ocho horas programadas y una utilización del turno de $75 \%$. La tabla 2 muestra la información de distancia y tiempos de ciclo, y la tabla 3 el dimensionamiento determinístico (analítico) para la flota de equipamientos.

Tabla 2. Distancia y tiempos de ciclo para los camiones

\begin{tabular}{lcc}
\hline \multicolumn{1}{c}{ Origen-destino } & $\begin{array}{c}\text { Distancia (2016) } \\
\text { km }\end{array}$ & $\begin{array}{c}\text { Tiempo de ciclo (2016) } \\
\text { minutos }\end{array}$ \\
\hline Mina Osvaldo-planta Ouro Branco (a) & 1,0 & 9,90 \\
\hline Mina Grande-planta Ouro Branco (a) & 1,0 & 9,94 \\
\hline Mina Mandacaru-planta Fillercal (b) & 1,8 & 13,53 \\
\hline Mina Antônio Ferreira-planta Fillercal (c) & 2,2 & 14,85 \\
\hline Mina Antônio Ferreira-Clasificación & 0,6 & 7,68 \\
\hline Clasificación-planta Fillercal (b) & 2,2 & 17,34 \\
\hline Planta Ouro Branco-pila de desmonte & 0,6 & 13,55 \\
\hline Pila de desmonte-mina Osvaldo & 1,4 & 3,25 \\
\hline Pila de desmonte-mina Grande & 1,4 & 3,16 \\
\hline
\end{tabular}

Elaboración propia 
Tabla 3. Dimensionamiento de flota de carguío y transporte

\begin{tabular}{|c|c|c|c|c|c|c|c|}
\hline & & M. Osvaldo & M. Grande & M. Ferreira & M. Mandacaru & Clasificación & $\begin{array}{c}\text { Pila de } \\
\text { desmonte }\end{array}$ \\
\hline \multirow{17}{*}{ 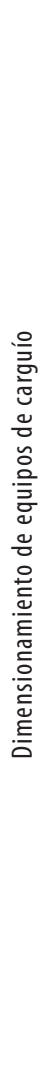 } & Modelo de excavadora & CAT336D & CAT336D & CAT336D & CAT336D & САТ950H & САТ950H \\
\hline & Horas por semana & 96 & 96 & 96 & 96 & 96 & 96 \\
\hline & Disponibilidad por turno & $80 \%$ & $80 \%$ & $80 \%$ & $80 \%$ & $80 \%$ & $80 \%$ \\
\hline & Horas efectivas por semana & 76,80 & 76,80 & 76,80 & 76,80 & 76,80 & 76,80 \\
\hline & Días por semana & 6 & 6 & 6 & 6 & 6 & 6 \\
\hline & Semanas por mes & 4 & 4 & 4 & 4 & 4 & 4 \\
\hline & Horas por mes & 307,20 & 307,20 & 307,20 & 307,20 & 307,20 & 307,20 \\
\hline & Capacidad de la cuchara $\left(\mathrm{m}^{3}\right)$ & 2,55 & 2,55 & 2,55 & 2,55 & 3,60 & 3,60 \\
\hline & Capacidad operacional & 2,295 & 2,295 & 2,295 & 2,295 & 3,240 & 3,240 \\
\hline & Factor de llenado & $90 \%$ & $90 \%$ & $90 \%$ & $90 \%$ & $90 \%$ & $90 \%$ \\
\hline & Peso específico esponjado $\left(\mathrm{t} / \mathrm{m}^{3}\right)$ & 1,50 & 1,50 & 1,50 & 1,50 & 1,50 & 1,50 \\
\hline & Tiempo de ciclo (min.) & 0,28 & 0,28 & 0,28 & 0,28 & 0,53 & 0,53 \\
\hline & Factor de eficiencia & $72 \%$ & $72 \%$ & $72 \%$ & $72 \%$ & $72 \%$ & $72 \%$ \\
\hline & Disponibilidad mecánica & $90 \%$ & $90 \%$ & $90 \%$ & $90 \%$ & $90 \%$ & $90 \%$ \\
\hline & Rendimiento (t/h) & 531,13 & 531,13 & 531,13 & 531,13 & 396,14 & 396,14 \\
\hline & Producción por día (t) & 625 & 625 & 780 & 188 & 780 & 417 \\
\hline & Número de excavadoras & 0,09 & 0,09 & 0,13 & 0,03 & 0,15 & 0,08 \\
\hline \multirow{8}{*}{ 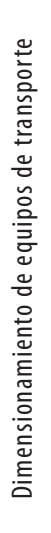 } & Modelo de camión / volquete & VM330 & VM330 & VM330 & VM330 & VM330 & VM330 \\
\hline & Circuito & 1 & 2 & 3 & 4 & 5 & 6 \\
\hline & Horas por semana & 96 & 96 & 96 & 96 & 96 & 96 \\
\hline & Disponibilidad por turno & $80 \%$ & $80 \%$ & $80 \%$ & $80 \%$ & $80 \%$ & $80 \%$ \\
\hline & Horas efectivas por semana & 76,80 & 76,80 & 76,80 & 76,80 & 76,80 & 76,80 \\
\hline & Días trabajados por semana & 6 & 6 & 6 & 6 & 6 & 6 \\
\hline & Semanas por mes & 4 & 4 & 4 & 4 & 4 & 4 \\
\hline & Horas trabajadas por mes & 307,20 & 307,20 & 307,20 & 307,20 & 307,20 & 307,20 \\
\hline
\end{tabular}


(continuación)

\begin{tabular}{lcccccc}
\hline Capacidad de la tolva $\left(\mathrm{m}^{3}\right)$ & 24 & 24 & 24 & 24 & 24 & 24 \\
\hline Capacidad operacional & 19,2 & 19,2 & 19,2 & 19,2 & 19,2 & 19,2 \\
\hline Factor de llenado & $80 \%$ & $90 \%$ & $90 \%$ & $90 \%$ & $90 \%$ & $90 \%$ \\
\hline Peso específico esponjado $\left(\mathrm{t} / \mathrm{m}^{3}\right)$ & 1,50 & 1,50 & 1,50 & 1,50 & 1,50 & 1,50 \\
\hline Tiempo de ciclo (min.) & 10,0 & 13,5 & 13,5 & 15,0 & 8,0 & 17,5 \\
\hline Factor de eficiencia & $72 \%$ & $72 \%$ & $72 \%$ & $72 \%$ & $72 \%$ & $72 \%$ \\
\hline Disponibilidad mecánica & $90 \%$ & $90 \%$ & $90 \%$ & $90 \%$ & $90 \%$ & $90 \%$ \\
\hline Rendimiento (t/h) & 124,42 & 92,16 & 92,16 & 82,94 & 155,52 & 71,09 \\
\hline Producción por día (t) & 1250 & 417 & 188 & 125 & 780 & 780 \\
\hline Número de camiones & 0,80 & 0,40 & 0,20 & 0,10 & 0,40 & 0,90 \\
\hline
\end{tabular}

Circuito 1: Osvaldo, Grande-Ouro Branco; circuito 2: Ouro Branco-pila de desmonte; circuito 3: Mandacaru-Fillercal; circuito 4: A. Ferreira-Fillercal; circuito 5: A. Ferreira-Clasificación; circuito 6: clasificación-Fillercal.

Fuente: datos facilitados por la Gerencia del Grupo Pirineus

Elaboración propia

\subsection{Dimensionamiento estocástico de la flota de carguío y transporte en la mina \\ Ouro Branco}

Cabral (2010), sostiene que un modelo de simulación permite expresar problemas complejos y de carácter dinámico. Noronha (2016) menciona los cinco pasos que debe seguir un estudio de investigación de operaciones: definición del problema, construcción del modelo, solución del modelo, validación e implementación. Para la mina en estudio los tiempos establecidos se muestran en la tabla 4.

Tabla 4. Tiempo empleado por los equipos de carguío y transporte

\begin{tabular}{l|l|l}
\hline Dominio & Origen-destino & Tiempo \\
& & tiempo de viaje vacío \\
& tiempo de carguío \\
& & tiempo de viaje cargado \\
& tiempo de descarga \\
\hline
\end{tabular}


(continuación)

\begin{tabular}{|c|c|c|}
\hline \multirow[t]{4}{*}{ Zona 1} & Clasificación-planta Fillercal & $\begin{array}{l}3,59+1,24 * \operatorname{BETA}(1,58,1,08) \\
\text { NORM }(5,73,0,263) \\
4,63+0,85^{*} \text { BETA }(1,33,1,24) \\
0,15+1,24 * \operatorname{BETA}(1,75,1,92)\end{array}$ \\
\hline & Antônio Ferreira-planta Fillercal & $\begin{array}{l}\text { a) } 3,76+\operatorname{LOGN}(0,369,0,255)+\operatorname{LOGN}(0,369,0,255) \\
\text { b) } 2,42+1,46^{*} \mathrm{BETA}(1,99,1,67) \\
\text { c) } \operatorname{NORM}(5,32,0,419) \\
\text { d) } 0,15+1,24^{*} \mathrm{BETA}(1,75,1,92)\end{array}$ \\
\hline & Antônio Ferreira-clasificación & $\begin{array}{l}\text { a) } 0,93+\operatorname{WEIB}(0,31,2,08) \\
\text { b) } 2,42+1,46^{*} \operatorname{BETA}(1,99,1,67) \\
\text { c) } 1+0,7 * \operatorname{BETA}(2,08,1,82) \\
\text { d) TRIA }(0,34,0,634,0,76)\end{array}$ \\
\hline & Mandacaru-planta Fillercal & $\begin{array}{l}\text { a) } 3,84+\operatorname{WEIB}(0,522,2,72)+\operatorname{LOGN}(0,218,0,154) \\
\text { b) } 2,31+1,03 * \operatorname{BETA}(2,1,52) \\
\text { c) } \operatorname{NORM}(4,6,0,244) \\
\text { d) } 0,15+1,24 * \operatorname{BETA}(1,75,1,92)\end{array}$ \\
\hline \multirow[t]{3}{*}{ Zona 2} & Osvaldo-planta Ouro Branco & $\begin{array}{l}\text { a) } 1,99+\operatorname{GAMM}(0,227,3,09)+\operatorname{ERLA}(0,0728,3) \\
\text { b) } 1,56+\operatorname{GAMM}(0,305,4,4) \\
\text { c) } 2,18+\operatorname{LOGN}(0,478,0,373) \\
\text { d) } \operatorname{TRIA}(0,48,0,8,1,12)\end{array}$ \\
\hline & M. Grande-planta en Ouro Branco & $\begin{array}{l}\text { a) } \operatorname{TRIA}(1,61,1,67,3)+\operatorname{TRIA}(0,63,0,915,1,2) \\
\text { b) } 2+1,7^{*} B E T A(1,15,1,24) \\
\text { c) } 2,18+\operatorname{LOGN}(0,478,0,373) \\
\text { d) TRIA }(0,48,0,8,1,12)\end{array}$ \\
\hline & $\begin{array}{l}\text { Pila de desmonte en planta-pila de } \\
\text { desmonte }\end{array}$ & $\begin{array}{l}\text { a) } 1+0,69^{*} \operatorname{BETA}(1,66,179)+\operatorname{TRIA}(0,49,0,835,1,18) \\
\text { a1) } 0,5+\operatorname{NORM}(3,25,0,376)+\operatorname{ERLA}(0,0728,3) \\
\text { a2) } 2,54+1,21^{*} \operatorname{BETA}(2,04,1,93)+\operatorname{TRIA}(0,63,0,915,1,2) \\
\text { b) } \operatorname{NORM}(6,12,0,187) \\
\text { c) } 1,13+\operatorname{ERLA}(0,12,5) \\
\text { d) } 0,29+0,43^{*} \operatorname{BETA}(1,4,1,32)+\operatorname{TRIA}(0,57,0,815,1,06) \\
1 \text { Pila de desmonte a Osvaldo. } \\
2 \text { Pila de desmonte a M. Grande }\end{array}$ \\
\hline
\end{tabular}

Fuente: Funciones de tiempo encontradas con el software Input Analyzer ARENA 14, a partir de una base de 25 datos para cada una de las actividades que componen el ciclo de cada operación. Tales informaciones representan medias poblacionales (aplicación y conceptos de teoría de límite central) y fueron tomadas en el año 2016 en campo. 
Para la implementación del modelo que simula las operaciones de carguío y transporte en la mina Caliza Ouro Branco fue necesario realizar el Diagrama de Ciclo de Actividades (DCA) de producción (figura 2 ) de la mina en estudio.

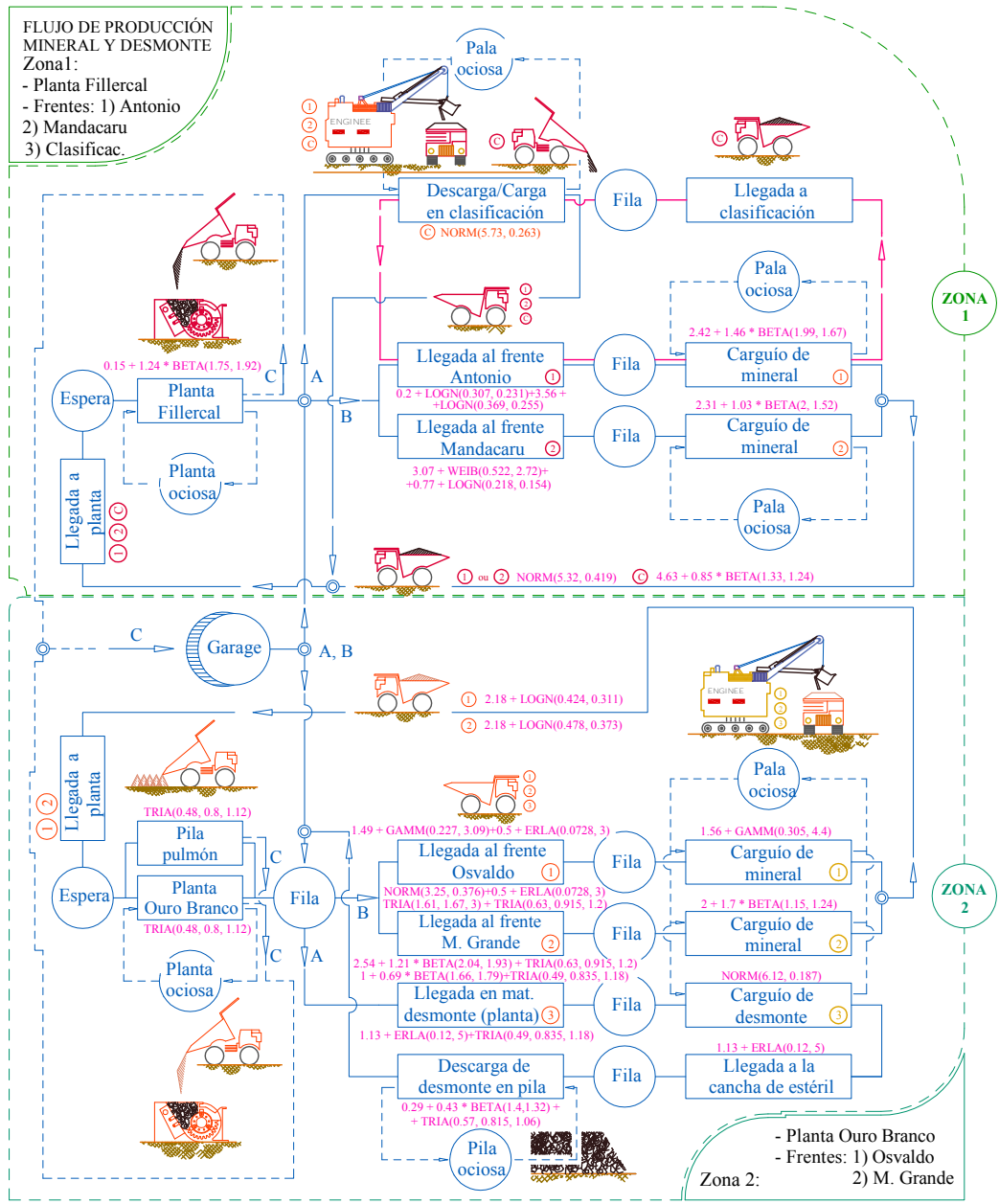

Entidades permanentes: pala, volquete, chancadora, pila

\section{ATRIBUTOS:}

Capacidad del volquete: $28,8 \mathrm{t}$

Volquete trabajando en clasificación $(\odot$

N. ${ }^{\circ}$ volquetes para Fillercal: 03

N. ${ }^{\circ}$ volquetes para Ouro Branco: 02

Turno de trabajo $(\mathrm{t})=16$ horas/dia $(12 \mathrm{HET})$
DESVIACIONES:

$\mathrm{t}=\mathrm{t}-1$

A, B : $t>0$

$C: t=0$

Figura 2. Diagrama de ciclo de actividades de producción mineral en la mina Ouro Branco

Elaboración propia 
También se desarrolló el modelo de simulación de las operaciones de carguío y transporte como se muestra en la figura 3.

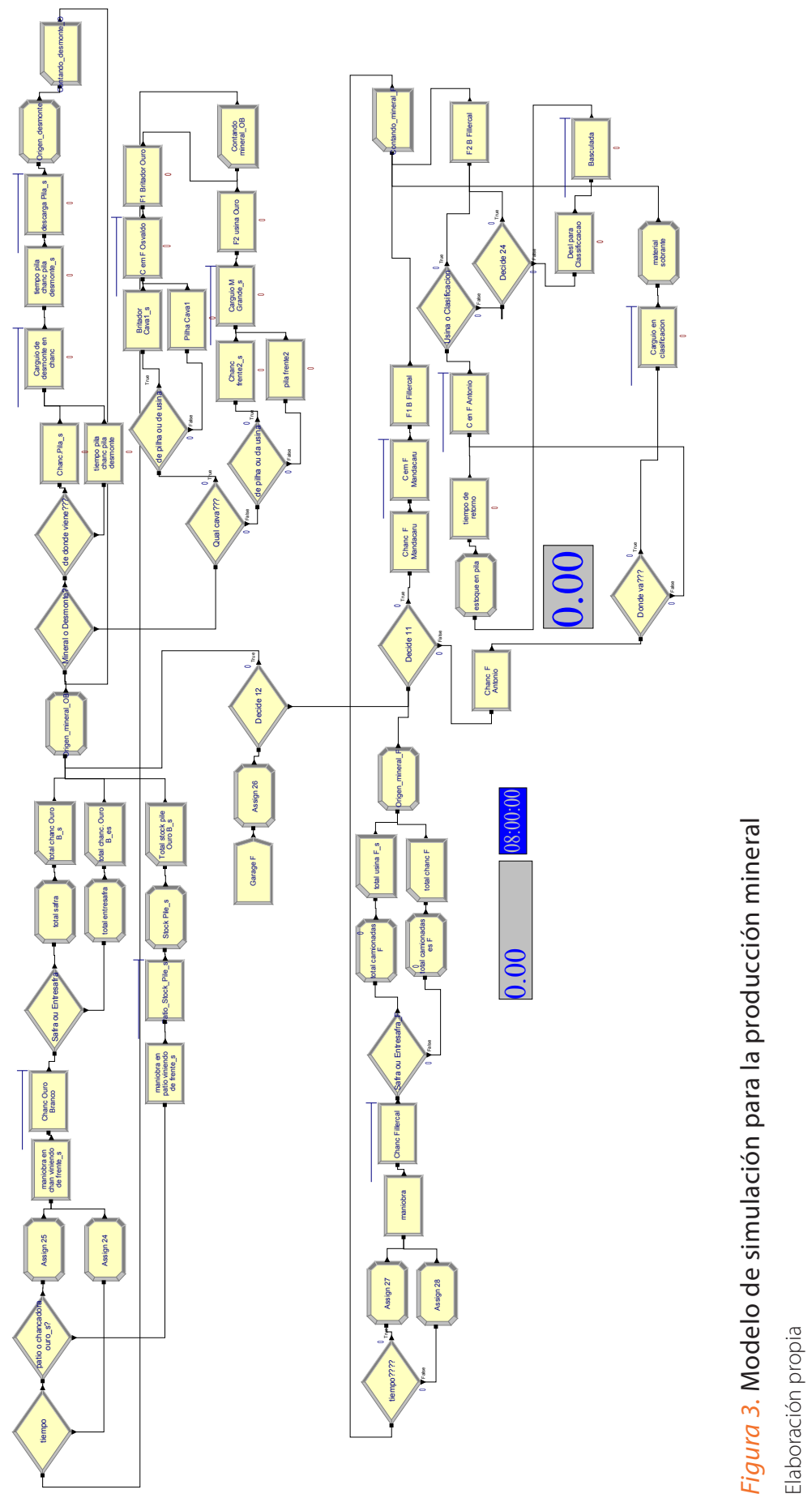


El modelo de dimensionamiento de carguío y transporte representado en la figura 3 está expresado en el software ARENA 14 (desarrollado inicialmente por Systems Modeling en 1993, como sucesor de SIMAN y CINEMA, que fueron presentados en 1982 y 1984 respectivamente). En el citado modelo son usados módulos integrados de simulación (CREATE, ASSIGN, DECIDE, RECORD, PROCESS) para eventos discretos, los cuales expresan las actividades desarrolladas por las unidades (camión) y realizadas por los recursos (excavadora). Tales unidades y recursos realizan actividades (carga, transporte, descarga, espera) definidas y condicionadas por expresiones de variable aleatoria, que es definida por un requerimiento de producción. En la tabla 5 se muestran los resultados obtenidos en ARENA 14.

Tabla 5. Resultados de la simulación para la flota de carguío y transporte

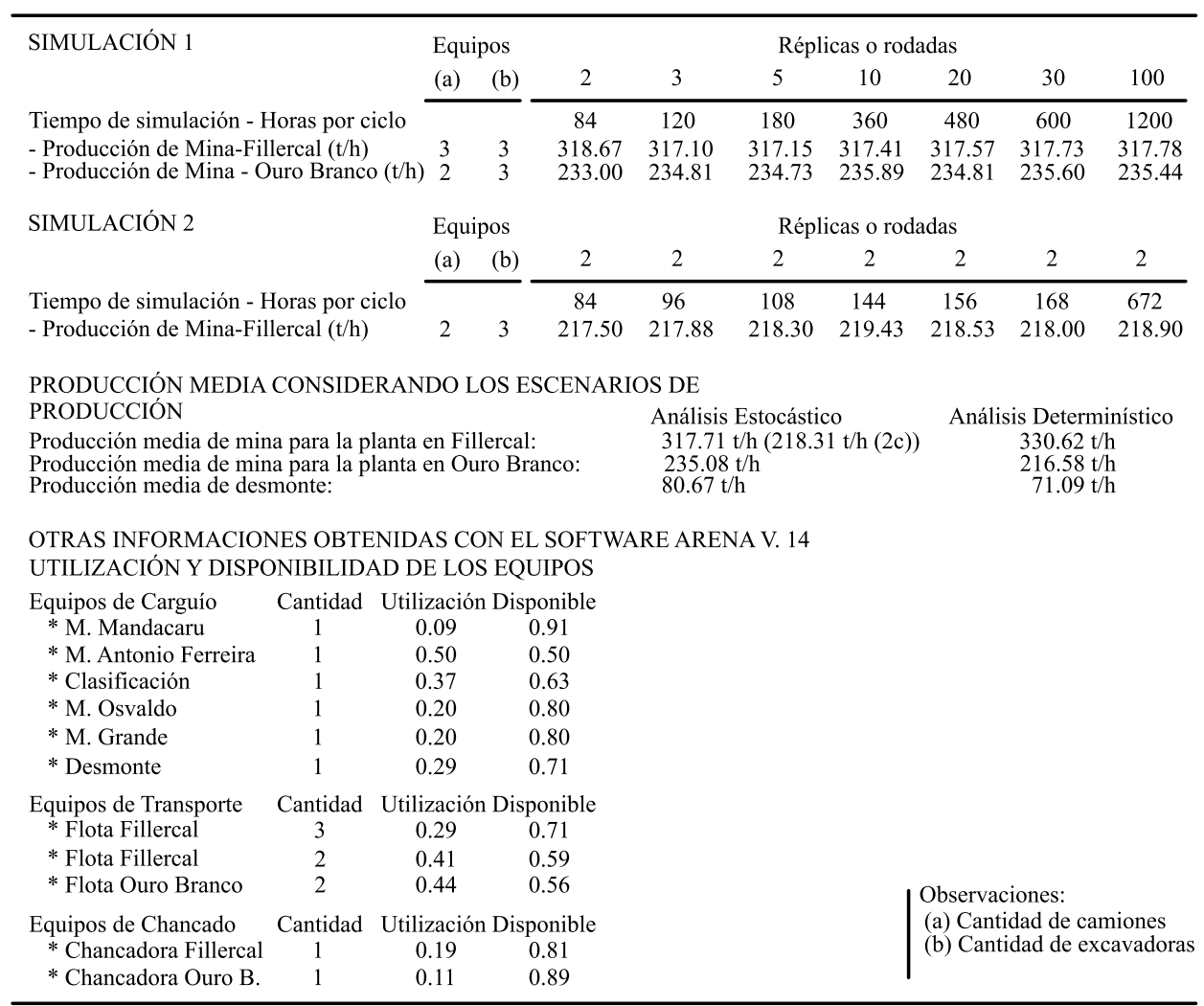

Elaboración propia 


\section{Conclusiones}

A través de la simulación se pueden elaborar modelos que representen de manera más precisa el modelo real de producción, cuyo propósito es predecir el comportamiento de las entidades y analizar los resultados. Además, el modelo permite la visualización del proceso de manera sistemática, integrada y unificada, facilitando el entendimiento y la evaluación del comportamiento de las operaciones ya mencionadas en varios escenarios.

Las conclusiones más importantes a partir del estudio comparativo son las siguientes:

i) En la simulación se establecieron variaciones en los tiempos y ciclos de rodada, y los resultados de estos escenarios permitieron ajustar la producción programada deseada (tabla 5). Los tiempos empleados en realizar cada actividad que compone el ciclo de producción de los equipamientos de carguío y transporte para el dimensionamiento analítico y estocástico están determinados por cantidades definidas como valor (tabla 3) y funciones de probabilidad (tabla 4). A pesar de que los tiempos de ciclo son diferentes, la producción de la flota determinada con ambos métodos es consistente (tabla 5).

ii) Al analizar el cálculo de dimensionamiento analítico (determinístico) y la simulación con base en la meta de producción para el año 2017, se concluye que es necesario el uso de seis excavadoras (cuatro CAT 336D y dos CAT 950H para el carguío de mineral y desmonte). Por otro lado, conforme a los resultados obtenidos en la tabla 5, la meta puede ser alcanzada con cuatro camiones: dos para atender la producción de la planta en Fillercal y dos para Ouro Branco. Finalmente, la producción horaria estimada para el dimensionamiento analítico de la planta en Fillercal es igual a 330,62 t/h, y para la planta en Ouro Branco, $216,58 \mathrm{t} / \mathrm{h}$. En cuanto al dimensionamiento determinístico para la planta en Fillercal es igual a 317,71 t/h, y para la planta en Ouro Branco 235,08 t/h (tabla 5).

iii) Los índices de utilización de los equipos de carguío están relacionados con la cantidad de material producido (requerido). En el dimensionamiento convencional, al considerar los cuatro camiones, el índice de utilización es de $15 \%$ en clasificación, $8 \%$ en chancadora, $9 \%$ en las minas Osvaldo y Grande, 3 \% en Mandacaru y 13 en Antônio Ferreira. Mientras que en el dimensionamiento con simulación con cuatro camiones se tiene $25 \%$ en la clasificación, $28 \%$ en la chancadora, $20 \%$ en las Minas Osvaldo y Grande, $7 \%$ en la Mandacaru y $35 \%$ en Antônio Ferreira. Considerando cinco camiones, se tiene $37 \%$ en la clasificación, $28 \%$ en la chancadora, $20 \%$ en las Minas Osvaldo y Grande, $9 \%$ en Mandacaru y 50 \% en Antônio Ferreira. En el dimensionamiento convencional las tasas de utilización de las excavadoras son calculadas de acuerdo con la producción requerida; ya que en la simulación es el software el que calcula la capacidad de producción con el uso de cuatro y cinco camiones. 


\section{Referencias}

Arena (2007). Arena ${ }^{\circledR}$ User's Guide. Rockwell Automation. Estados Unidos de América.

Bise, C. (2003). Minning Engineering Analysis. Society for Mining, Metallurgy, and Exploration, Inc (SME).

Cabral, I. E. (2011). Simulacao de operacoes mineiras. Notas de Aula, Programa de Pós-graduação em Engenharia Mineral, PPGEM - Escolas de Minas, Universidade Federal de Ouro Preto.

Dessureault, S. (2009). Operational equipment technology. Arizona: Mining and Geological Engineering, University of Arizona.

Koppe, J. (2007). A lavra e a indústria mineral no Brasil. Estado da arte e tendências tecnológicas. En: Fernandes, F.; Castilhos, Z.; Luz, A. B. y Matos, G. (eds.), Tendências - Brasil 2015 - Geociências e Tecnologia Mineral, Parte II - Tecnologia Mineral, Rio de Janeiro: CETEM-Centro de Tecnologia Mineral.

Noronha, O. (2016). Simulação e dimensionamento de frota das operações de carregamento e transporte na mineração Calcário Ouro Branco Ltda. Goiás: Escola de Minas da Universidade Federal de Goiás.

Nuñez, A. E. C. (2011). Dimensionamento de Equipamentos de Lavra - Apostila. Departamento de Engenharia de Minas. Universidade Federal de Goiás. Goiás: Catalão, Notas de Aula.

Pinto, L. R. y Saliby, E. (1999). Sistema para simulação a eventos discretos utilizando Borland Deplhi. III Simpósio de Pesquisa Operacional e Logística da Marinha (SIMIN).

Silva, V. C. (2009). Carregamento e transporte de rochas. Ouro Preto: Escola de Minas da Universidade Federal de Ouro Preto.

Sweigard, R. J. (1992). Materials handling: Loading and Haulage. Hartman, H. L. Society for Mining, Metallutgy, and Exploration, Mining engineering handbook. 2a edition, volume one. Littleton, Colorado: SME. 
